\title{
Prospective « Compétitivité des oléagineux dans l'avenir »
}

\section{Future study "The oilseed competitiveness in the future"}

Oléagineux, Corps Gras, Lipides. Volume 9, Numéro 5, 362-8, Septembre - Octobre 2002, Dossier : Prospective et recherche agronomique

\begin{abstract}
Auteur(s) : Clementina SEBILLOTTE, Antoine MESSEAN, Laurent RUCK, INRA, Délégation permanente à l'agriculture, au développement et à la prospective (DADP), 147, rue de l'Université, 75338 Paris cedex 07, France.
\end{abstract}

Author(s) : Clementina SEBILLOTTE, Antoine MESSEAN, Laurent RUCK

Résumé : La prospective a pour mission d'éclairer les décisions en explorant, de façon collective, le champ des futurs possibles et en dégageant différents scénarios pour l'avenir. Le CETIOM a choisi cette démarche pour étudier la compétitivité des oléagineux (colza et tournesol) en France en tenant compte du contexte européen et mondial à l'horizon 2010-2015. La concurrence entre les oléagineux métropolitains et les autres sources de corps gras s'exerce à tous les niveaux : production, transformation, consommation... Aussi, cette démarche prospective a mobilisé plus de nombreuses personnes de différentes disciplines, activités économiques et institutions. La méthode de prospective utilisée, développée à la DADP-INRA, est fondée sur une vision systémique du problème et fait appel aux modèles de représentation graphique. À l'issue de ce travail, sept dimensions stratégiques ont été retenues : * les agricultures et leurs relations avec leur environnement économique ; * la place de l'information dans la consommation des corps gras ; * la prise en compte des recommandations nutritionnelles par les industries de transformation ; ${ }^{*}$ I'évolution des modèles d'alimentation animale ; ${ }^{*}$ la place des oléagineux dans les systèmes de production ; ${ }^{*}$ les oléagineux face au défi de l'environnement ; * l'impact des usages non alimentaires dans le développement des oléagineux. Pour chacune de ces sept dimensions, des microscénarios de futurs possibles ont été élaborés. Cet article présente brièvement les principaux traits des microscénarios de chacune de ces sept dimensions stratégiques.

Summary : The objective of future studies is to enlighten decision-makers, by exploring collectively the sphere of possible futures and bringing out different scenarios for the future. CETIOM has carried out such an approach in order to assess the competitiveness of oilseed (rapeseed and sunflower) in France and Europe, taking into account the European and world-wide context by 2010-2015. The competition between domestic oilseed crops and other sources of fats takes place at all levels of the production, transformation and consumption processes. Thus, this future study has involved about 150 people from several scientific fields, economic bodies and institutions. The overall foresight methodology used has been designed by DADP-INRA and is based on a systemic view of the various issues addressed and requires graphic representation models. Seven strategic topics were accepted at the end of this work: * agriculture and its relationship with its economic environment; * the role of information on fat consumption; * the consideration of nutritional recommendations made by the transformation industries; ${ }^{*}$ the evolution of animal feeding models; ${ }^{*}$ the place of oilseed crops 
within production systems; * oilseed crops and the environmental challenge; ${ }^{*}$ the impact of nonfood uses in oilseed development. For each of these seven topics, various micro-scenarios have been drawn up. The micro-scenarios of each one of these seven strategic topics are briefly described in this article.

Mots-clés : prospective, microscénarios, axe stratégique, oléagineux, compétitivité.

Keywords : foresight, future prospect, future study, micro-scenario, strategic topic, oilseeds, competitiveness.

\section{ARTICLE}

\section{Pourquoi une prospective au CETIOM ?}

L'évolution de notre contexte - la mondialisation des marchés associée à une plus forte segmentation, la globalisation des enjeux, l'industrialisation accrue de l'agriculture, l'apparition de nouvelles exigences vis-à-vis de la qualité des produits mais aussi vis-à-vis de leurs modes de production, l'émergence de nouveaux acteurs et de nouvelles solidarités entre la ville et la campagne, la multiplication des centres de décision, les perspectives ouvertes par l'explosion des nouvelles technologies - se traduit en fait par une montée de l'incertitude et une complexité croissante des problèmes et des questions à traiter.

Le progrès des sciences et des technologies élargit considérablement le champ des applications possibles. De la prise de conscience que tout progrès scientifique n'est pas nécessairement bon ou utile et du fait même de la sophistication des technologies, il résulte des exigences accrues en termes de sécurité alimentaire, de transparence, d'information et de contrôle de ces applications.

Face à l'évolution de ce contexte, les acteurs de la recherche et du développement, et en particulier les instituts techniques, doivent adapter leur positionnement stratégique, leurs métiers et leurs programmes. Il est alors indispensable d'anticiper les évolutions futures afin de mieux s'y préparer, voire même de les orienter. C'est le sens de la démarche prospective qui a pour mission d'éclairer les décisions d'aujourd'hui en explorant, de façon collective, le champ des futurs possibles.

Le but de cette prospective entreprise par le CETIOM sur la compétitivité des oléagineux en France, compte tenu du contexte européen et mondial est alors, à partir d'une meilleure connaissance du système oléagineux dans son ensemble, des acteurs et de leurs enjeux, des évolutions futures des techniques et des technologies comme des conditions socio-économiques et de politiques agricoles, d'éclairer les décideurs en dégageant différents scénarios pour le futur, à l'horizon 2010-2015.

\section{Une démarche collective}

La réflexion prospective menée par le CETIOM sur la compétitivité des oléagineux, a associé de nombreux agents du CETIOM, de I'INRA et des personnalités extérieures et a bénéficié de l'appui méthodologique de la DADP ${ }^{2}$ à I'INRA. Elle a mobilisé un groupe de référence constitué de 18 
personnes issues du CETIOM, de la filière et d'autres organismes, et quatre groupes d'experts qui ont rassemblé environ 120 participants.

Le groupe de référence était constitué de : M. Baron (Limagrain), A. Brinon (Saipol et Fedhuil'), G. Clerjaud (Cavac), O. de Gasquet (Fop), B. de Verneuil (Fop et CETIOM), Y. Dronne (INRA), J. Évrard (CETIOM), E. Foncelle (Fop et CETIOM), J.-L. Gurtler (Sido puis Oniol), J.-L. Lespinas (CETIOM), A. Messéan ${ }^{3}$ (CETIOM), J.-M. Meynard (INRA), E. Pilorgé (CETIOM), M. Renard (INRA), L. Ruck ${ }^{4}$ (CETIOM), C. Sebillotte ${ }^{5}$ (CETIOM), M. Sebillotte ${ }^{6}$ (INRA-DADP) et G. Vermeersch (Sofiproteol). Le rôle du groupe de référence était de proposer des orientations techniques de travail ; d'apporter une partie des informations; de constituer un premier niveau de synthèse et de validation des résultats ; de constituer une première phase de valorisation des travaux. En son sein un noyau permanent a assuré l'animation et la coordination ainsi que la production des travaux et l'application des orientations méthodologiques.

Selon la méthode de prospective SYSPAHMM, développée à la DADP (INRA), décrite par ailleurs dans ce dossier, la première étape du travail a consisté à représenter graphiquement le système oléagineux et de décrire son fonctionnement actuel à travers les processus qui expliquent sa dynamique. « De qui et de quoi parle-t-on, jusqu'à quel degré de précision et comment en parle-t-on ? (...) Puis nous avons décrit le fonctionnement actuel de ce système (aspect dynamique) en analysant ses processus, chacun se résumant sous la forme d'une phrase. Ces processus expliquent ce qui se passe dans le système, ce que les acteurs font " (Sebillotte C. 2000).

Ensuite, le groupe de référence a élaboré un ensemble d'hypothèses qui portent sur tous les compartiments du système et sur leurs relations.

À cette étape du travail, les résultats intermédiaires ont été soumis à des experts extérieurs pour approfondir certaines questions, compléter et valider la première représentation du système proposé et l'ensemble des hypothèses, et enfin légitimer la démarche. Quatre groupes d'experts de différentes disciplines et de différentes institutions ont été réunis à deux occasions autour de quatre domaines d'évolution : dans la production agricole ; dans les industries de transformation ; dans l'alimentation animale ; dans les métiers du conseil. Parallèlement, des experts ont été consultés individuellement.

Une fois validée la liste d'hypothèses retenues (105 dans notre cas), le groupe de référence les a fait interagir pour analyser les influences qu'elles ont les unes sur les autres et les a ensuite combinées afin de construire des microscénarios organisés autour de sept dimensions stratégiques. Ces microscénarios sont décrits en détail dans la suite de cet article.

Après l'élaboration de ces microscénarios décrivant des futurs possibles, nous entrons pleinement dans la valorisation des résultats de la prospective. Des stratégies potentielles pour la filière oléagineuse et le CETIOM ainsi qu'une première déclinaison régionale ont, d'ores et déjà, été proposées à titre d'exemple dans le rapport de cette prospective. Mais la valorisation passe d'abord par l'appropriation des microscénarios par les décideurs à différents niveaux, au sein du CETIOM, de la filière, de l'INRA et des différents partenaires concernés afin d'en faire un véritable outil d'aide à la décision. À cette fin, les principaux défis auxquels est aujourd'hui confronté le secteur oléagineux vont être analysés avec la grille d'analyse que constitue cette prospective : la révision à mi-parcours de la PAC, la place des oléagineux sur le marché des protéines, la segmentation des débouchés, les 
nouveaux partenariats à développer et la contribution des oléagineux au développement durable constituent quelques-uns de ces questionnements actuels. La déclinaison régionale de cette réflexion, déjà engagée par le CETIOM, fait de la prospective un outil d'animation et de réflexion en partenariat étroit avec les acteurs locaux.

Par ailleurs, le groupe " recherche oléagineux " de I'INRA, auquel participe le CETIOM, élabore, à partir des résultats de cette prospective, des propositions de stratégies de recherche pour les oléagineux. Enfin, les évolutions du contexte socio-économique ainsi que du système oléagineux luimême imposent une actualisation du jeu d'hypothèses que nous avons utilisées et nous instaurons une veille collective, en partenariat (CETIOM-INRA) pour permettre d'actualiser et de maintenir ce cadre de réflexion stratégique.

\section{Des microscénarios autour de sept dimensions stratégiques}

Si les résultats de cette prospective ne peuvent pas se réduire à la production des microscénarios, ceux-ci en constituent cependant une partie importante. Les microscénarios sont des récits de futurs possibles qui abordent un secteur particulier de préoccupations (c'est pourquoi nous les appelons microscénarios et non scénarios). Le groupe de référence de la prospective "Compétitivité des oléagineux dans l'avenir » les a élaborés suivant la méthode SYSPAHMM conçue par la DADP (INRA) et qui est décrite dans d'autres articles de ce même numéro ${ }^{7}$.

Les sept dimensions stratégiques autour desquelles ont été construits les microscénarios ne constituent pas une entrée prédéterminée, mais une sortie du travail. Elles résultent des relations que le groupe a établies entre les hypothèses et du regroupement des hypothèses les plus liées entre elles.

Si chaque dimension stratégique est plus centrée sur un problème particulier et concerne plus fortement certains compartiments du système oléagineux, on trouve en son sein des hypothèses appartenant à presque tous les compartiments du système. II s'agit de focaliser sur un problème sans perdre de vue l'esprit systémique de la démarche.

Les sept dimensions stratégiques obtenues sont :

* Les agricultures et leurs relations avec leur environnement économique ;

* la place de l'information dans la consommation des corps gras ;

* la prise en compte des recommandations nutritionnelles par les industries de transformation ;

* l'évolution des modèles d'alimentation animale ;

* la place des oléagineux dans les systèmes de production ;

* les oléagineux face au défi de l'environnement ;

* I'impact des usages non alimentaires dans le développement des oléagineux.

Autour de chacune de ces dimensions, des microscénarios de futurs possibles ont été construits. 
Décrivons, brièvement ces sept groupes de microscénarios.

\section{Le groupe de microscénarios 1 : Les agricultures et leurs relations avec leur environnement économique}

L'agrégat qui est à l'origine de ces microscénarios est constitué de deux sous-ensembles d'hypothèses, le premier, plus lié à l'agriculture, le second, plus lié à l'industrie.

La construction des cinq microscénarios s'organise autour d'une hypothèse motrice selon laquelle : « En UE, le poids de l'aval sur son amont, en particulier celui de la grande distribution, se renforce de plus en plus (cahiers des charges pour la production et la transformation, etc.). »

Ce moteur principal se combine avec différents moteurs auxiliaires, variables selon les microscénarios, qui permettent de construire cinq microscénarios se différenciant par l'acteur majeur qui les oriente. Ainsi, dans le microscénario msc 1.1 " Agriculture d'entreprise ", l'acteur prépondérant est l'agriculteur en tant que producteur agricole mais aussi comme entrepreneur et gestionnaire de son entreprise ; dans le msc 1.2, les acteurs dominants appartiennent à l'aval de la production agricole (grande distribution, industrie de transformation) ; dans le microscénario msc 1.3, c'est l'industrie de trituration qui structure la filière oléagineuse ; dans le microscénario msc 1.4, ce sont les organismes d'approvisionnement et de collecte qui ont le rôle principal tandis que dans le microscénario msc 1.5 c'est le monde agricole dans sa dimension collective qui est le protagoniste (schéma 1).

Le contexte de ces microscénarios est celui d'une Europe qui maintient des versements compensatoires aux exploitations agricoles.

Dans le premier microscénario msc 1.1 " Agriculture d'entreprise ", les exploitations agricoles recherchent la rentabilité maximale des capitaux investis, en particulier, à travers une diminution de l'ensemble des charges. Ayant cet objectif, les agriculteurs agrandissent leurs exploitations ou/et multiplient les formes d'association permettant une gestion commune du travail et des matériels, ce qui diminue les charges fixes à l'hectare. Ils utilisent de plus en plus d'outils d'aide à la décision pour mieux gérer leurs grandes exploitations et maintenir des niveaux de production qui assurent la rentabilité maximale des capitaux investis. Ils nouent de nouvelles relations en termes d'information, de conseil, de commerce. Dans ce contexte les $\mathrm{OS}^{8}$ ont un rôle de moins en moins central face à l'apparition de nouveaux acteurs spécialisés avec lesquels ces relations se nouent.

Dans le microscénario msc 1.2 " Une agriculture dominée par l'aval ", les agriculteurs cherchent à augmenter la marge nette de leurs exploitations surtout à travers une augmentation du produit brut. Pour cela, ils passent des contrats avec la grande distribution et avec l'industrie. L'agriculteur devient un " technicien exécutant " des cahiers des charges imposés et conçus par l'aval. Dans ce microscénario, le marketing de l'aval conditionne fortement l'innovation agricole et les OS ont un rôle moins central dans les contrats et le conseil et se concentrent sur la vente de produits aux agriculteurs et de services à l'aval.

Le microscénario msc 1.3 « L'industrie de trituration structure la filière oléagineuse » est une variante du microscénario antérieur : dans ce cas c'est l'industrie de trituration qui s'occupe, pour les 
oléagineux, de la collecte, la commercialisation et l'établissement de contrats, la transformation, l'exportation... assurant ainsi un approvisionnement local et tracé, répondant à ses besoins de qualité. Le rôle d'intermédiation des organismes de collecte est très affaibli pour les oléagineux.

Dans le microscénario msc 1.4 " Le rôle majeur des organismes d'approvisionnement et de collecte ", ce sont les OS qui structurent l'activité agro-économique, créent des districts agro-industriels spécialisés et coordonnent les entreprises impliquées. Les OS renforcent leurs rôles d'intermédiation entre production-transformation et distribution, de services aux agriculteurs, d'approvisionnementcollecte, de conseil... Ils développent une segmentation des productions et garantissent l'industrie et la grande distribution d'un approvisionnement en matières premières, de qualité et tracées.

Dans le microscénario msc 1.5 "Le projet du monde agricole de gestion collective de l'espace rural », le monde agricole propose des projets locaux de gestion de l'espace rural avec le concours de la « société ". La profession agricole en assure l'animation et le développement, et crée un espace de dialogue entre les acteurs ruraux, urbains et les pouvoirs publics. II y a rénovation du contrat entre l'agriculture et la société avec de nouveaux objectifs.

\section{Le groupe de microscénarios 2 : La place de l'information dans la consommation des corps gras}

Dans cet agrégat de 15 hypothèses, on considère que le moteur général est l'hypothèse : « À côté du prix et de son revenu, le consommateur européen (et des pays industrialisés) intègre de plus en plus des facteurs informationnels (nutritionnels, de santé, environnementaux, de traçabilité...) et le facteur marque dans ses achats. "Dans un premier temps (2000-2008), ces différents facteurs ont une influence plus ou moins forte sur les choix des consommateurs, mais aucun de ces facteurs ne prime clairement sur les autres dans leurs décisions d'achat. Dans un deuxième temps (2008-2015), les facteurs informationnels sont déterminants pour le consommateur. On identifie alors trois microscénarios (schéma 2).

Dans le premier, msc 2.1.1, les besoins nutritionnels déterminent les choix alimentaires des consommateurs. Le deuxième, msc 2.1.2, est une variante dans laquelle les consommateurs se nourrissent en suivant les indications de tables d'alimentation et en respectant leurs besoins nutritionnels individuels. Enfin, dans le troisième, msc 2.2, les choix alimentaires des consommateurs sont guidés par le souci de disposer de produits authentiques et liés au territoire, les besoins nutritionnels passant au second plan.

\section{Le groupe de microscénarios 3 : La prise en compte des recommandations nutritionnelles par les industries de transformation (huile)}

Ce groupe de microscénarios 3 se situe dans la poursuite du raisonnement de deux des trois microscénarios du groupe 2 dans lesquels l'information nutritionnelle détermine la consommation des corps gras.

Il existe deux débouchés pour les huiles : I'alimentaire et le non-alimentaire. Pour chacun, il existe deux manières d'obtenir ces huiles : soit en sélectionnant des variétés adaptées grâce aux progrès de la génétique et en produisant des graines fournissant des huiles ayant des profils d'acides gras « spécifiques ", c'est-à-dire répondant directement aux besoins (quitte à ce que ces profils, entre autres pour l'industrie non alimentaire, soient mono-acides gras), soit en procédant à des mélanges d'huiles et dans ce cas le rôle de la génétique et de l'amélioration des plantes peut se trouver 
restreint, ou uniquement orienté à la production d'espèces mono-acide gras. Pour l'industrie non alimentaire, des études de débouchés permettent de connaître les besoins les plus probables, en revanche la satisfaction des besoins des industries de l'alimentation humaine dépend des recommandations des nutritionnistes. Or la consommation alimentaire représente environ $80 \%$ de la consommation totale mondiale des corps gras, aussi décide-t-on que le moteur de cet agrégat est I'hypothèse : "Les recommandations des nutritionnistes évoluent. " On entend par là que les recommandations des nutritionnistes, même s'il n'y a pas aujourd'hui de controverse fondamentale dans le monde à ce sujet, sont encore instables sur certains points et donc susceptibles d'évoluer dans les années à venir, vers de nouvelles recommandations, atténuant, complétant, voire contredisant les recommandations anciennes.

Couplé à deux moteurs auxiliaires qui concernent le comportement des industries de l'huile et I'utilisation des OGM, deux groupes de microscénarios sont élaborés. Un premier groupe (les trois microscénarios 3.1, schéma 3) correspond à une situation dans laquelle les recommandations nutritionnelles sont très évolutives. Pour suivre cette évolution, dans un premier temps, l'utilisation des huiles combinées (mélanges d'huiles) se généralise. Dans un deuxième temps, les industries de l'agroalimentaire prennent en compte les recommandations nutritionnelles. Dans msc 3.1.1, les huiles de table et les produits fabriqués par les industries agro-alimentaires sont chacun équilibrés nutritionnellement, dans le msc 3.1.2, les industries de l'huile et les industries agro-alimentaires produisent des produits qui sont complémentaires dans le domaine de la nutrition lipidique pour permettre au consommateur de se " construire " une diète équilibrée tout en conservant le plaisir de manger, dans le msc 3.1.3, les industries de l'huile produisent des huiles combinées à partir de mélanges d'acides gras extraits des huiles.

Le deuxième groupe (les six microscénarios 3.2, schéma 4) correspond à une situation dans laquelle les recommandations nutritionnelles sont peu évolutives, ce qui permet à l'industrie de continuer à proposer des huiles génériques. Ce groupe rassemble six microscénarios dans lesquels les produits proposés par les industries de l'huile et de l'agro-alimentaire varient selon l'interprétation que feront les consommateurs des recommandations nutritionnelles lipidiques; interprétation influencée par les médias, la publicité, les groupes de pression industriels et scientifiques, les modes et les idées reçues. Dans le msc 3.2.1, la consommation de l'acide linoléique est mise en cause, dans le msc 3.2.2 I'acide linolénique devient incontournable, dans le msc 3.2.3, c'est l'huile d'olive qui est préférée, dans le msc 3.2.4, domine la préoccupation de respecter le rapport oméga 6/oméga 3, dans les mcs 3.2.5 et 3.2.6 ce sont, respectivement, I'huile de colza et de soja qui s'imposent.

\section{Le groupe de microscénarios 4 « L'évolution des modèles d'alimentation animale "}

Les microscénarios du groupe 4 "L'évolution des modèles d'alimentation animale " ont déjà été examinés dans l'article de Sebillotte C. "Les microscénarios et leur construction. " Un exemple sur les microscénarios de l'axe stratégique " alimentation animale " de la prospective "Compétitivité des oléagineux dans l'avenir » dans ce même numéro ; nous n'y revenons pas.

\section{Le groupe de microscénarios 5 : La place des oléagineux dans les assolements}

Le contexte de ce microscénario est celui d'une Europe où les rapports de prix de vente entre cultures pourront subir des variations conjoncturelles mais qui, de manière générale, ne s'installeront pas définitivement en faveur d'une espèce plus que d'une autre. Par ailleurs, l'Union 
européenne maintient des versements compensatoires aux exploitations agricoles découplés des cultures.

On décide, alors, que le moteur de cet agrégat de 15 hypothèses est l'ensemble formé par les trois hypothèses qui concernent soit les améliorations de rendement et de réduction de coût de production des variétés de colza et de tournesol, soit les évolutions de rendement du colza ou du tournesol par rapport aux céréales.

Le moteur général de ce groupe de microscénarios se résume ainsi : les rendements des oléagineux (colza et tournesol) augmentent, deviennent plus réguliers (tournesol), et leurs coûts de production diminuent par rapport aux céréales, grâce aux résultats des recherches génétiques, agronomiques et technico-économiques et à leur mise en œuvre par les agriculteurs.

À circonstances socio-économiques voisines et avec des relations de prix stables en moyenne, la compétitivité des cultures, reflétée dans les microscénarios du groupe 5 par l'importance des surfaces dans les assolements, dépend assez directement des productions à l'hectare, donc des rendements et de leur régularité, et de la diminution des coûts de production. On ne fait pas jouer dans ce groupe de microscénarios les avantages environnementaux des oléagineux, traités dans le groupe de microscénarios 6 , ni les effets d'une segmentation plus poussée par la qualité, traités également ailleurs. Enfin, selon les microscénarios de ce groupe 5, ce moteur peut se doubler d'un moteur auxiliaire représenté par l'hypothèse qui dit que les agriculteurs diversifient leurs activités productives pour diminuer les risques économiques liés à la variabilité des prix et des rendements. Cette hypothèse peut aussi largement contribuer, dans les exploitations de grande culture, au maintien des oléagineux dans les assolements, même si les progrès de rendement de ceux-ci sont plus modestes ; la diversification consiste, ici, à ne pas opter pour des assolements dominés par la monoculture.

Les six microscénarios, construits à partir des moteurs mentionnés ci-dessus, se différencient par les "progrès " techniques réalisés sur les différents systèmes de cultures et les conséquences qui en résultent sur les assolements (schéma 5 ). Ainsi, dans le microscénario msc 5.1 les progrès techniques réalisés sur les systèmes de cultures avec colza et tournesol ont comme conséquence un développement de ces cultures dans les assolements. Dans le msc 5.2, les améliorations obtenues sur la culture de colza se traduisent par une augmentation de sa surface dans les assolements. En ce qui concerne le msc 5.3, l'absence de progrès techniques sur les cultures oléagineuses fait qu'elles ne restent que comme têtes de rotations dans les systèmes de culture. Pour le msc 5.4 , les progrès techniques faits sur les rotations blé sur blé en continu et l'absence d'améliorations techniques dans les systèmes de culture avec oléagineux conduit à la quasi-disparition des oléagineux dans les assolements. Dans le msc 5.5 , les progrès réalisés sur le soja permettent d'introduire de manière plus importante cette culture dans les assolements européens. Enfin, dans le msc 5.6, les améliorations réalisées sur les protéagineux métropolitains (pois d'hiver et de printemps, féverole, lupin) permettent d'augmenter les rendements de ces cultures et leur compétitivité face aux autres têtes d'assolement et aux céréales. L'intérêt de ces microscénarios est de faire réfléchir, aux côtés des recherches classiques de génétique et d'amélioration des plantes, à l'importance de travaux agronomiques et technico-économiques sur les techniques culturales et sur les conditions organisationnelles qui jouent sur les coûts de production unitaires et sur la régularité des 
rendements. Ces microscénarios soulignent aussi l'intérêt de recherches sur l'appréciation des risques économiques par les acteurs et sur leurs comportements face à ces risques.

Pour les pays de l'UE et des PECO, les surfaces en cultures oléoprotéagineuses concernant chacun de ces microscénarios ont été estimées de manière semi-quantitative, en confrontant dires d'experts et informations bibliographiques aux éléments statistiques actuels et historiques. Ainsi, par exemple, dans l'Union européenne la surface de deux cultures, colza et tournesol, a été estimée pour le msc 5.1 " Développement des surfaces de colza et de tournesol " à environ près de 7,5 millions d'hectares, tandis que pour le msc 5.4 « Déclin des oléagineux en UE » l'estimation est d'un peu plus de 2 millions d'hectares.

\section{Le groupe de microscénarios 6 : Les oléagineux face au défi de l'environnement}

Dans un contexte de préoccupation de la société pour les questions d'environnement et d'occupation du territoire, considérée comme une tendance lourde à l'horizon de la prospective, on décide que le moteur de cet agrégat de 14 hypothèses est formé des deux hypothèses suivantes :

- À moyen terme, l'agriculture dans le monde sera totalement libéralisée (cours mondiaux, absence d'aides et de prix d'intervention).

- La politique agricole européenne favorise l'émergence d'une agriculture territoriale et durable (préservation de l'environnement, du paysage, occupation du territoire... ; elle appuie la politique française des contrats de production liés au territoire).

À partir de ces moteurs, trois microscénarios ont été élaborés (schéma 6). Dans le premier, msc 6.1 « Les oléagineux dans l'agriculture durable ", la politique agricole reconnaît un intérêt environnemental à la culture des oléagineux et les favorise en considérant leur culture comme une des pratiques donnant droit aux aides qui répondent à des critères d'écoconditionalité.

Dans le deuxième microscénario, msc 6.2 " Les oléagineux et les coûts environnementaux ", la politique agricole ne reconnaît pas d'intérêt environnemental aux oléagineux. Elle verse des aides qui ne répondent pas à des critères d'écoconditionalité, mais, en même temps, la pollution de l'environnement est fortement taxée. Ce sont alors le bilan économique des exploitations et les marchés qui reconnaissent un intérêt environnemental aux oléagineux car leur présence dans les successions culturales diminue la pollution environnementale et permet ainsi aux agriculteurs de payer moins de taxes, ce qui augmente la valeur ajoutée de leurs productions.

Dans le troisième microscénario, msc 6.3 , la politique agricole ne soutient pas les productions agricoles mais la pollution de l'environnement reste fortement taxée ; on distingue, alors, deux variantes. Dans la première (msc 6.3.1), l'agriculture de production résiste. Elle est prise en main par de nouvelles entreprises agricoles transnationales qui localisent les cultures selon leur compétitivité dans les différentes régions du monde, la distribution des oléagineux se réalise ainsi en fonction d'un compromis entre leur rentabilité et leur comportement environnemental. Dans la deuxième variante (msc 6.3.2), l'agriculture de production décline et avec elle les différentes cultures. Le nombre d'exploitations viables dédiées à l'agriculture " de production » diminue et certaines exploitations agricoles se livrent, totalement ou partiellement, à des activités connexes à l'agriculture (tourisme 
rural, séjours de retour à la nature). Ainsi l'agriculture se réoriente profondément vers de nouvelles vocations (territoire, qualité, authenticité, paysage...). Les exploitations agricoles qui restent attachées à la production agricole réalisent une production à haute valeur ajoutée avec une tendance à l'extensification.

\section{Le groupe de microscénarios 7 : L'impact des usages non alimentaires dans le développement des oléagineux}

On décide que le moteur principal, de l'agrégat qui est à l'origine de ces microscénarios, est I'hypothèse : " L'utilisation de biocarburants et de biolubrifiants (à partir d'huiles végétales) augmentera en UE. " Sachant qu'on a admis que " la société est toujours préoccupée par l'environnement à l'horizon de la prospective ", ce qui renforce l'hypothèse motrice. Dans ce contexte, deux forces, l'État et les citoyens, orientent le fonctionnement du moteur et définissent les différents microscénarios :

- l'État par ses interventions, ou non, sur les aspects d'environnement, de sécurité énergétique et de santé ;

- les citoyens par leurs pressions sur l'État, leurs attitudes face à l'environnement et leurs pratiques de consommation alimentaire et non alimentaire qui influent sur les stratégies des industriels.

À partir de cet ensemble moteur, nous avons décidé d'élaborer quatre microscénarios (schéma 7). Ils ont en commun le fait que la société soit, dans ces quatre situations, préoccupée par la dégradation de la qualité de l'environnement. Dans trois de ces microscénarios, 7.1.1, 7.1.2 et 7.3, les usages non alimentaires des huiles végétales augmentent, tandis que dans le microscénario 7.2 ces usages diminuent.

Le premier cas de figure correspond à une forte augmentation des utilisations non alimentaires des huiles végétales. Dans le microscénario msc 7.1.1, les pouvoirs publics européens favorisent l'utilisation des huiles végétales pour des usages non alimentaires dans le but de contribuer à la protection de l'environnement, mais sans mesures d'encouragement des cultures à destination non alimentaire sur le sol européen. De plus, les consommateurs privilégient la qualité et la traçabilité des produits alimentaires à celles des produits non alimentaires ce qui ne contribue pas forcément au développement des surfaces à destination du non-alimentaire, leur croissance étant alors seulement encouragée par l'augmentation des débouchés en non alimentaire.

Une variante est le microscénario msc 7.1.2 dans lequel les pouvoirs publiques, tout en encourageant ces utilisations des huiles végétales, se donnent les moyens de promouvoir les cultures oléagineuses à destination non alimentaire sur le sol européen pour assurer un approvisionnement national minimum pour les industries du non-alimentaire dans le but principal de diminuer la dépendance énergétique vis-à-vis des produits pétroliers.

Dans un deuxième cas de figure, représenté par le microscénario msc 7.3, l'augmentation des usages non alimentaires des huiles végétales est plus modérée que dans les deux situations précédentes. En l'absence d'une politique de protection de l'environnement, ce sont les citoyens qui prennent en main cette protection à travers leurs comportements quotidiens. La bonne image environnementale 
des produits non alimentaires élaborés à partir des huiles végétales est à l'origine de l'augmentation de leur consommation.

Enfin, dans le troisième cas de figure (microscénario msc 7.2), les huiles végétales perdent leur place comme matière première dans l'industrie du non-alimentaire. Les sources d'énergie se diversifient, les biocarburants élaborés à partir des huiles végétales deviennent minoritaires et les huiles végétales sont remplacées par du suif dans les cas où cela est possible.

Notes :

${ }^{1}$ Précédemment responsable de la prospective et de la socio-économie à la direction scientifique du Centre technique interprofessionnel des oléagineux métropolitains (CETIOM).

${ }^{2}$ Délégation permanente à l'Agriculture, au Développement et à la Prospective.

${ }^{3}$ Direction et responsabilité générale.

${ }^{4}$ Co-animation des travaux avec Clementina Sebillotte.

${ }^{5}$ Coordination générale et responsabilité des travaux.

${ }^{6}$ Appui méthodologique.

${ }^{7}$ Sebillotte M, Sebillotte $\mathrm{C}$. « Recherche finalisée, organisations et prospective. La méthode SYSPAHM ». Sebillotte C. « Les microscénarios et leur construction. Un exemple sur les microscénarios de l'axe stratégique " alimentation animale » de la prospective Compétitivité des oléagineux dans l'avenir.»

8 OS : organisme stockeur. II s'agit de coopératives ou de négociants qui s'occupent de l'approvisionnement et/ou de la collecte agricole.

\section{REFERENCES}

BIBLIOGRAPHIE

SEBILLOTTE C, RUCK L, MESSÉAN A (2002). Prospective compétitivité des oléagineux dans l'avenir. CETIOM, Paris.

SEBILLOTTE C en collaboration avec RUCK L (2000). Prospective compétitivité des oléagineux dans I'avenir. État du travail. Rencontres Annuelles du CETIOM. CETIOM. Paris. 


\section{Illustrations}

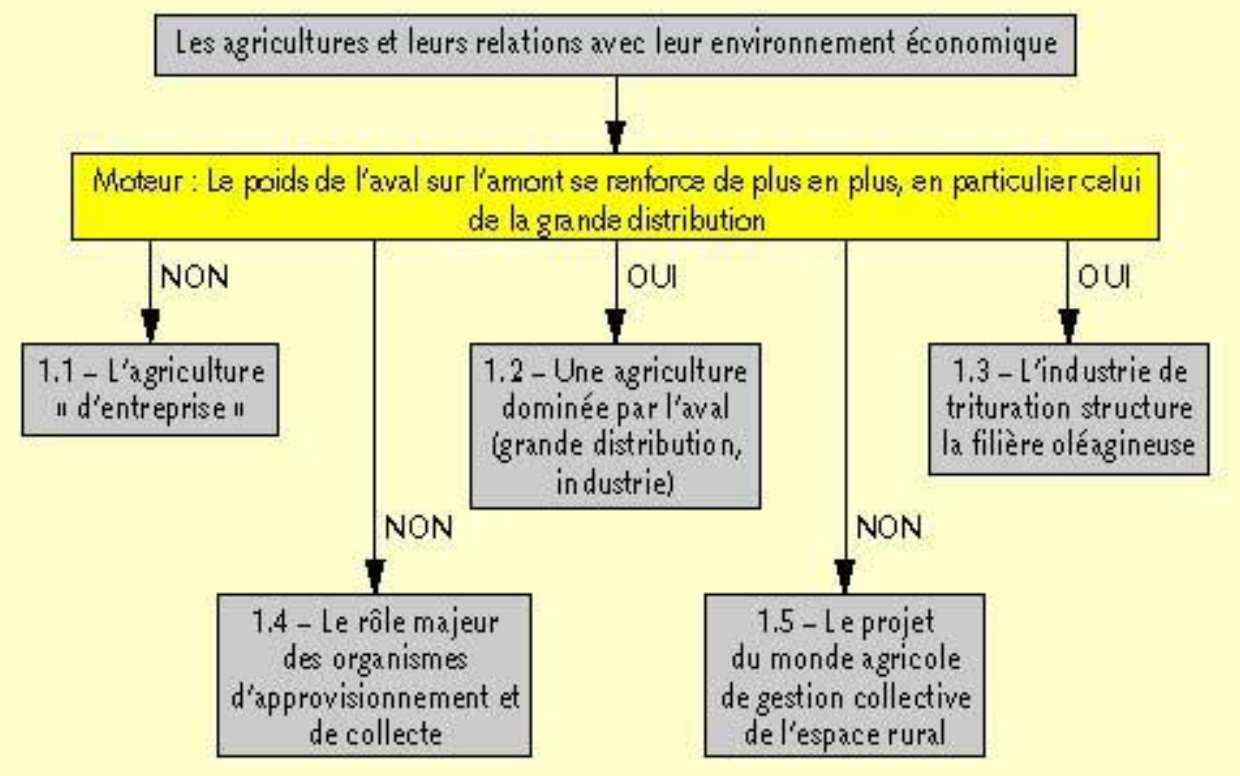

\section{Schéma 1. Groupe de microscénarios 1.}

\section{La place de l'information dans la consommation des corps gras}

Moteur : Les facteurs informationnels déteminent la consommation de corps gras

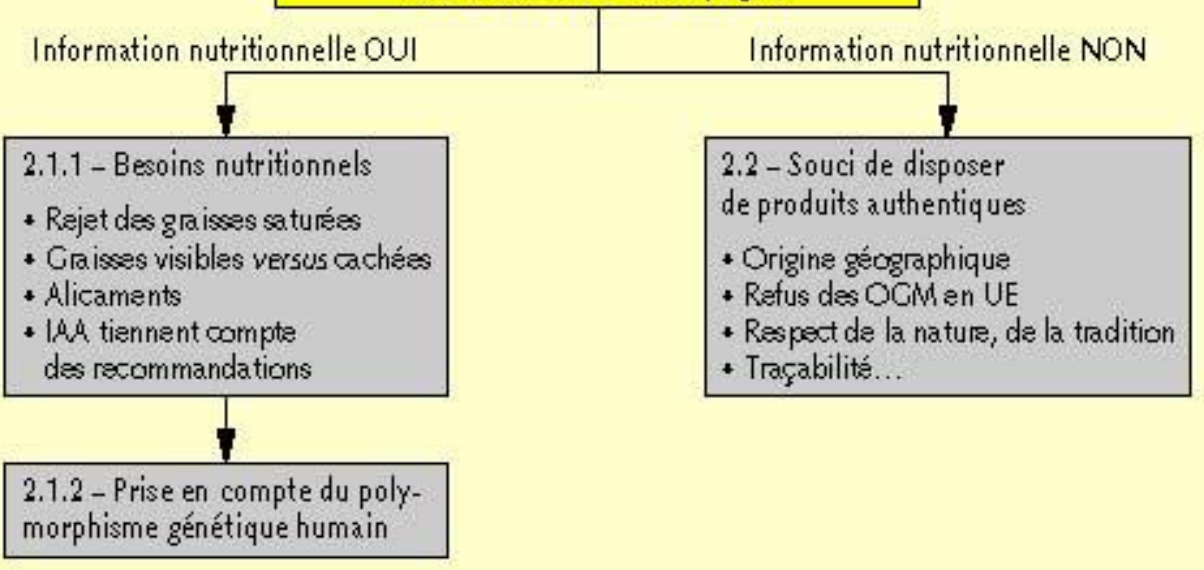

Schéma 2. Groupe de microscénarios 2 
Prise en compte des recommandations nutritionnelles par l'industrie de transformation

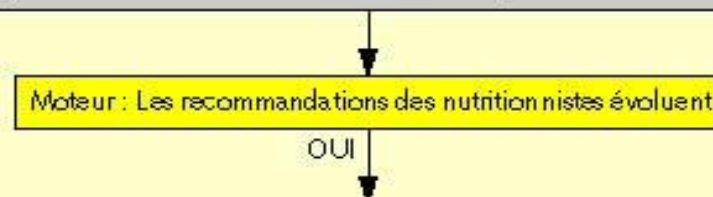

Dans un premier temps : Les huiles de table combinées comme conectrices.

Les IA,A continuent à utiliser toumesol, palme, colza...

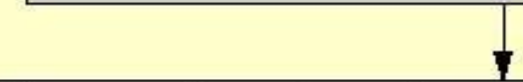

Dans un second temps: Le respect des recommandations nutritionnel les par industries de l'huile de table et par IAR

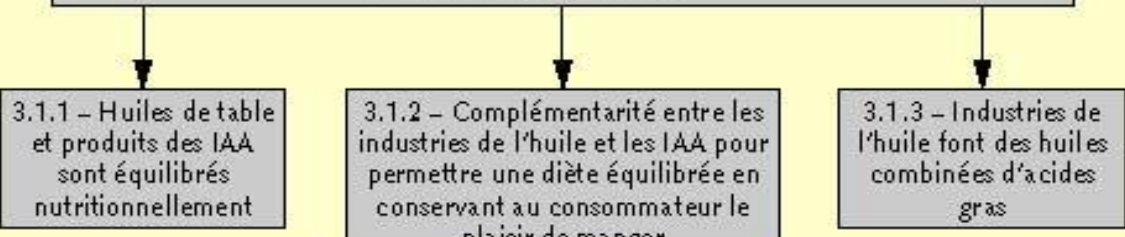

.Schéma 3. Groupe de microscénarios 3. Les microscénarios 3.1.

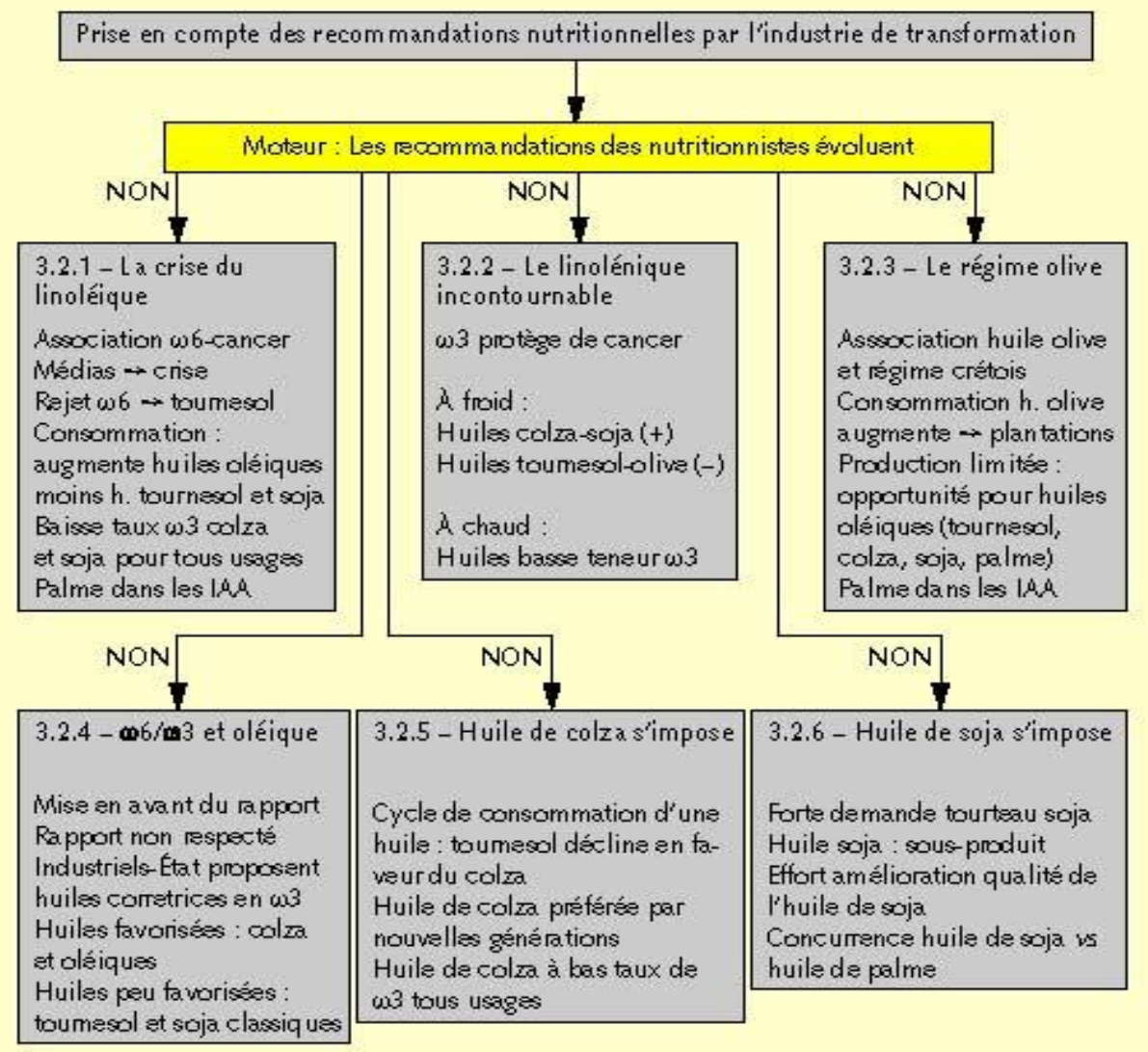

Schéma 4. Groupe de microscénarios 3. Les microscénarios 3.2. 


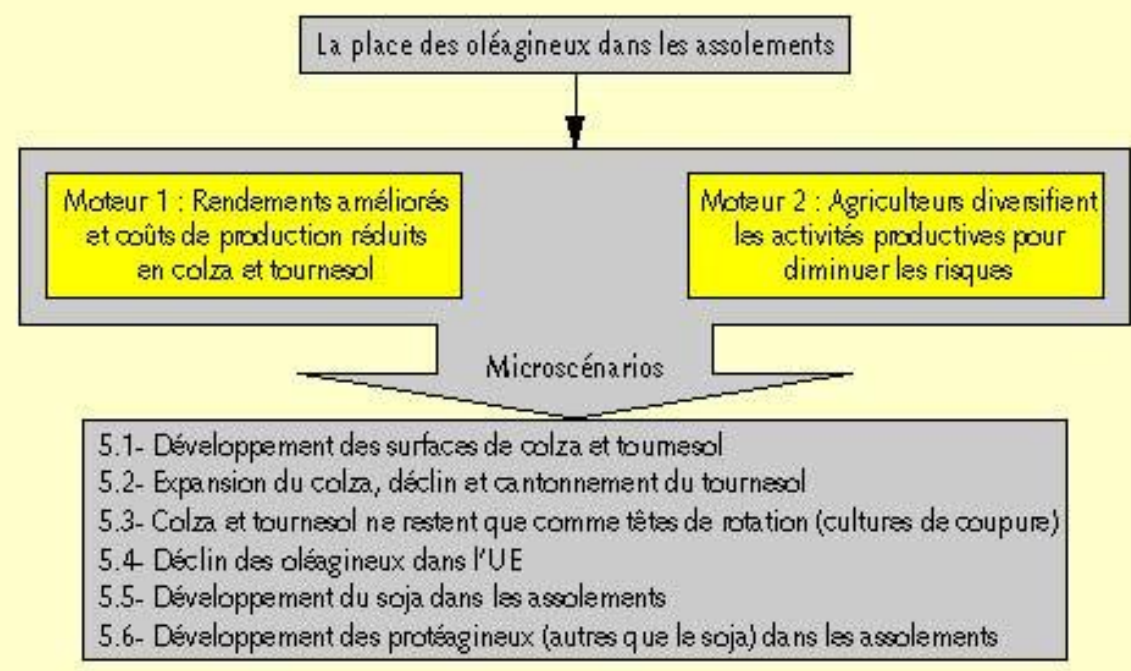

\section{Schéma 5. Groupe de microscénarios 5.}

\begin{tabular}{|c|c|c|}
\hline & \multicolumn{2}{|c|}{ Les oléagineux face au défi de l'environnement } \\
\hline \multicolumn{3}{|c|}{$\begin{array}{l}\text { Société próoccupée par en vironnement } \\
\text { Noteur } 1 \text { : L'agriculture dans le monde sera totalement libéralisée } \\
\text { Moteur } 2 \text { : La PAC favorise l'émergence d'une agriculture territoriale et durable }\end{array}$} \\
\hline $\begin{array}{r}\text { OUI/NON } \\
1 \text { OUI }\end{array}$ & $\begin{array}{r}\text { OUI/NON } \\
\text { /NON }\end{array}$ & $\begin{array}{r}\text { OUI/OUI } \\
\text { /NON }\end{array}$ \\
\hline $\begin{array}{l}\text { 6.1-Oléagineux } \\
\text { dansagriculture } \\
\text { durable } \\
\text { Aides aver } \\
\text { śo-conditionnalité }\end{array}$ & $\begin{array}{l}6.2 \text { - Oléagineux } \\
\text { et coûts } \\
\text { environnementaux } \\
\text { Aides sans } \\
\text { éco-conditionnalité }\end{array}$ & $\begin{array}{l}\text { 6.3 - Oléagineux et agriculture non soutenue } \\
\text { Sars aide à ta production } \\
\text { Pollution taxée } \\
\text { Panorama agricole UE change } \\
\text { Faible nombre d'exploitations agricoles viables }\end{array}$ \\
\hline $\begin{array}{l}\text { Oléagineux dans } \\
\text { successions : inté } \hat{e t} \\
\text { environnemental } \\
\text { reconnu } \\
\text { PÁC favorise les } \\
\text { oléagineux }\end{array}$ & $\begin{array}{l}\text { Pollution taxée } \\
\text { PAC ne favorise pas les } \\
\text { oléagineux } \\
\text { Oléagineux dars } \\
\text { successions : intérêt }\end{array}$ & \begin{tabular}{|l} 
6.3.1 - Résistance agriculture de production \\
Grandes exploitations ॥ industrielles et \\
trars-pays "I \\
Compromis rentabilitékenvironnement \\
Régionalisation des oléagineux
\end{tabular} \\
\hline & $\begin{array}{l}\text { emvionnemental } \\
\text { devientéconomique }\end{array}$ & $\begin{array}{l}\text { 6.3.2-Agriculture de production en déclin } \\
\text { Quelques exploitations agricoles extersives } \\
\text { à haute valeur ajouté } \\
\text { Exploitations agricoles : tourisme, paysage, santé } \\
\text { UE importatrice }\end{array}$ \\
\hline
\end{tabular}

Schéma 6. Groupe de microscénarios 6. 
Impact des usages non alimentaires dans le développement des oléagineux

Société prósccupée par en vironnement

Moteur : L'utilisation de biocarburants at de biolubrifiants augmente en UE

Intervention de l'état " pour " les huiles végétales en non alimentaire (NA)

Comportement des citoyens " pour " les huiles végétales en non alimentaire (NA)

\begin{tabular}{r|r|r|r|} 
OUI/OUI/ & OUI/OUI/ & OUI/NON & OUI/OUI/ \\
OUI/NON & OUI+/NON & NONONON & \\
\hline
\end{tabular}

7.1.1 - Usages NA des huiles végétales se développent

7.1 .2 - Usages $N A$ et développement des oléa gineux

- Politique favorable - Oléines UE - OCW interdits

$-\mathrm{C} 12, \mathrm{C} 14$ importés - Surface NA + ou =
- Politiqua favorable at recherche de sécurité energétique

- OCM acceptés

- C12, C14 en UE - Surface Na ++
7.2 - Déclin des utilisations NA des huiles végétales européennes

- Nouvelles techno $\log i \approx$

- Suit en NA

- C12, C14; OGM

- Surface Na = ou -
7.3 - Développement des usages NA porté par l'image des huiles végétales

- Actions citoyens pour environnement

- Pas de politique

- Surface Na + ou =

Schéma 7. Groupe de microscénarios 7. 